\title{
Effect of Satellite Motion on TWSTFT *
}

\author{
Sun Hongwei, Li Yuli, Yang Huae and Ding Xiaoqian \\ School of Mathematics and Computer Engineering Xi'an University of Arts and Science, \\ South Taibai Road 168, Xi'an, 710065, Shanxi Province, China \\ sunhongwei100@yahoo.com.cn
}

\begin{abstract}
The effect of the satellite motion on two-way satellite time and frequency transfer (TWSTFT) is analyzed. If two distances from two stations to the satellite are different, the non-reciprocal paths are produced, which impact the accuracy of TWSTFT. In addition, due to the Earth's rotation and the finite signal velocity, the non-reciprocal paths are also produced, and they mainly result from the variation of the satellite longitude and altitude.
\end{abstract}

Index Terms - Time transfer, Satellite, TWSTFT.

\section{Introduction}

Satellite is indispensable for time and frequency transfer of the highest precision and accuracy over large distances, because this cannot be achieved by terrestrial communication techniques. TWSTFT technique via the geo-stationary communication satellite is the greatest prospect in the remote and precise time and frequency transfer. Nowadays, several TWSTFT links in Asia-Pacific region have also been constructed using the communication satellite located $150^{\circ} \mathrm{E}$.

TWSTFT technique is for the point-to-point time transfer method. Each of the two laboratories requires a transmitting/receiving earth station to exchange timing information via a communication satellite, and the measurements consist of simultaneous time interval measurements at both sites in which the one pulse-per-second (1pps) signal is generated by the local clock. The principle of TWSTFT is set out in [1,2].

At present, the accuracy of the technique is towards as high as 1 nanosecond, therefore the impact of the systematic errors on accuracy must be studied in detail. A main factor affecting accuracy is satellite motion.

\section{Effect of satellite motion on accuracy}

The satellite used in TWSTFT is a geo-stationary satellite. Generally speaking, its motion mainly includes daily periodical motion and drift motion. To some extend, the distance from the station to the satellite can be considered as a sinusoid with amplitude and period of $24 \mathrm{~h}$ :

$$
R=R_{0}+R_{m} \cdot \sin (2 \pi t / 24+\phi)
$$

where $R_{0}$ is the normal distance from the station to the satellite, $R_{m}$ is the amplitude of the daily change, and $\square \square$ is the initial phase. The variation of the distance is:

$$
d R / d t=R_{m} \cdot \cos (2 \pi t / 24+\phi) \cdot \pi / 12
$$

The greatest rate of variation should be:

$$
d R / d t \leq R_{m} \cdot \pi / 12
$$

According to the various greatest amplitudes, we can get the greatest velocities of satellite motion relative the ground.

TABLE I Velocity of Satellite Relative Ground

\begin{tabular}{|c|c|}
\hline$R_{m}(\mathrm{~km})$ & $d R / d t \quad(\mathrm{~m} / \mathrm{s})$ \\
\hline 30 & $<2.18$ \\
\hline 60 & $<4.36$ \\
\hline 90 & $<6.54$ \\
\hline
\end{tabular}

Errors, relating to two aspects, result from the satellite motion. Since the two distances from two stations to the satellite are different, the time taken by the two signals from two stations to reach the satellite is different. Thus the satellite receives the two signals at different positions, which is shown in Fig. 1 (where G1 and G2 denote the two stations, and S1 and $\mathrm{S} 2$ denote the positions of the satellite).

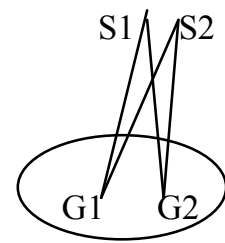

Fig1. The satellite receiving signal at different positions

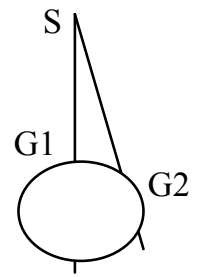

Fig.2 Greatest distance difference from two stations to satellite

An extreme case is with one station having an elevation of $6^{\circ}$ and the other having an elevation of $90^{\circ}$. Thus the greatest difference from the two stations to the satellite is $5893 \mathrm{~km}$

\footnotetext{
* This work is supported by Natural Science Basic Research Plan in Shaanxi Province (No. 2011JM1011) and by Science and Technology Plan Projects of Xian (No. CXY1134WL37, No. CX12189WL08 and No. CX12189WL22) of China.
} 
(see Fig. 2). The corresponding greatest time difference is $19.66 \mathrm{~ms}$. If the greatest amplitude reaches $60 \mathrm{~km}$, according to the greatest velocity of the satellite motion relative the station, the non-reciprocal error is $<290 \mathrm{ps}$.

Table II shows such results of the non-reciprocities in some links that include TL-NICT, KRISS-NICT, SPRINGNICT, and NTSC-NICT, where NICT is National Institute of Information and Communications Technology of Japan, TL is Telecommunication Laboratories of Taiwan, KRISS is Korea Research Institute of Standards and Science, SPRING is Productivity and Standards Board of Singapore and NISC is National Time Service Center of China. It is easy to conclude when choosing a satellite for which both elevations are about the same, which can minimize the effect; or the effect can be eliminated by offsetting the transmission time.

TABLE II Non-reciprocities Due to Different Distance

\begin{tabular}{|l|c|c|}
\hline \multicolumn{1}{|c|}{ Links } & Different distance [km] & $\begin{array}{c}\text { Non-reciprocities[ps] } \\
\text { (amplitude: } 60 \mathrm{~km})\end{array}$ \\
\hline TL-NICT & 31 & $<1.6$ \\
\hline KRISS-NICT & 441 & $<27.4$ \\
\hline SPRING-NICT & 745 & $<36$ \\
\hline NTSC-NICT & 1238 & $<60$ \\
\hline
\end{tabular}

Due to the Earth's rotation and the finite signal velocity, the non-reciprocal paths are produced. The effect on TWSTFT is called Sagnac effect, which can be calculated by following formula [3].

$$
E=2 \omega A / C^{2}
$$

where $\square$ is the rate of Earth's rotation, $C$ is the velocity of light, $A$ is the equatorial projection of the area of the quadrangle the vertices of which are the center of the Earth and the positions of the stations on the surface of the Earth, and the position of the satellite with respect to the Earth's surface. It is thus a function of the positions of two stations and the satellite. The extreme case is about $420 \mathrm{~ns}$ for two stations at the equator, with each having an elevation of $6^{\circ}$, causing a maximal separation in longitude.

For the link of NTSC-NICT, the value of the Sagnac effect is $85.7 \mathrm{~ns}$, and the effect coefficients of longitude, latitude and altitude of the satellite variation are approximately $\quad 66 \mathrm{ps} / 0.1^{\circ}, \quad 0.13 \mathrm{ps} / 0.1^{\circ}$ and $2 \mathrm{ps} / \mathrm{km}$, respectively. Table III shows such results of the nonreciprocities due to the Sagnac effect in some links. It shows that the errors primarily depend on the variation of the satellite longitude and altitude. Based on the extent of the satellite motion from the satellite control center (the longitude is within $150^{\circ} \mathrm{E} \pm 0.05^{\circ}$, the latitude is within $0^{\circ} \pm 0.05^{\circ}$, and the aviation of altitude is $<60 \mathrm{~km}$ ), the error from the Sagnac effect is at order of $100 \mathrm{ps}$ in several links.

TABLE III Non-reciprocities Due to Sagnac Effect

\begin{tabular}{|l|c|c|c|c|}
\hline \multicolumn{1}{|c|}{ Links } & Sagnac effect $[\mathrm{ns}]$ & $\begin{array}{c}\text { Variation from longitude } \\
{\left[\sim \mathrm{ps} / 0.1^{\circ}\right]}\end{array}$ & $\begin{array}{c}\text { Variation from latitude } \\
{\left[\sim \mathrm{ps} / 0.1^{\circ}\right]}\end{array}$ & $\begin{array}{c}\text { Variation from altitude } \\
{[\sim \mathrm{ps} / \mathrm{km}]}\end{array}$ \\
\hline TL-NICT & 63.83 & 2 & 0.10 & 0.6 \\
\hline KRISS-NICT & 34.54 & 23 & 0.05 & 3.0 \\
\hline PSB-NICT & 125.13 & 40 & 0.20 & 2.0 \\
\hline NTSC-NICT & 85.70 & 66 & 0.13 & 0.8 \\
\hline
\end{tabular}

\section{Conclusions}

TWSTFT, employing a commercial communication satellite, is the only used technique allowing a remote timetransfer with accuracy potential being below 1ns. A main factor affecting the accuracy is the satellite motion. Errors, of two aspects, result from the satellite motion. One is that since the two distances from two stations to the satellite are different, the satellite receives the two signals at different position, and the non-reciprocities are produced. The largest non-reciprocal error is less than 50 ps in the links of AsiaPacific region. Another is that due to the Earth's rotation and the finite signal velocity, the non-reciprocal paths are also produced. We established that the variation primarily results from the satellite longitude and altitude, and the error from the Sagnac effect, is at order of 100 ps in these links.

\section{References}

[1] J. A. Davis, "European two-way satellite time transfer experiments using the INTELSAT satellite at 307E," IEEE Transactions on Instrumentation and Measurement, vol. 44, no. 2, pp. 90-93, Feb. 1995.

[2] Michto Imae, "Two-way satellite time and frequency transfer network in pacific rim region," IEEE Transactions on Instrumentation and Measurement, vol. 50, no. 2, pp. 559-562, Feb. 2001.

[3] E. J. Post, "Sagnac effect," Reviews of Modern Physics, vol. 39, no. 2, pp. $475-493$, Feb. 1967 